Doug Geisler, Eva K. Grebel, and Dante Minniti, eds.

\title{
Testing Intermediate-age Stellar Evolution with Magellanic Cloud Clusters
}

\author{
Carme Gallart \\ Andes Prize Fellow. Departamento de Astronomía, Univ. de Chile, and \\ Department of Astronomy, Yale Univ., USA
}

Manuela Zoccali

European Southern Observatory, Germany.

Giampaolo Bertelli

National Council of Research, Italy

Cesare Chiosi

Dipartimento di Astronomia dell'Università di Padova, Italy

Pierre Demarque

Department of Astronomy, Yale University, USA.

Leo Girardi

Dipartimento di Astronomia dell'Università di Padova, Italy

Sukyoung Yi

California Institute of Technology, USA

\begin{abstract}
We present new deep VLT color-magnitude diagrams of three intermediate-age populous star clusters in the LMC. These data will be used independently by the Padova and Yale stellar evolution Groups to address issues relevant to the evolution of intermediate-mass stars: mainly the extent of the core convective overshoot, as well as other poorly determined parameters, such as mass loss during stellar evolution, internal rotation, and He content.
\end{abstract}

\section{Introduction}

The Magellanic Clouds' (MC) star clusters offer an exceptional opportunity to study stellar evolution. Populous star clusters of a wide variety of ages and chemical compositions can be observed at effectively the same distance. They contain young metal-poor stellar populations that are absent in the Galaxy. These will be particularly valuable in interpreting stellar populations in high 
and intermediate redshift galaxies, and in studying the star formation history of nearby dwarf galaxies from photometry of their resolved stars.

We obtained high quality photometry of a sample of MC clusters with FORS at the VLT, in a range of ages to constrain intermediate-age stellar evolution models. Stellar evolution problems that we want to address include establishing the extent of the core convective overshoot and helping to disentangle the relative importance of other poorly determined parameters, such as mass loss during stellar evolution, internal rotation, and He content. These data will be used independently by two stellar evolution groups, the Padova Group (Chiosi, Bertelli, Girardi) and the Yale Group (Demarque, Yi).

Here we present color-magnitude diagrams reaching well below the main sequence turnoff of the intermediate-age (2-4 Gyr) LMC clusters NGC 2155, NGC 2173 and SL556. An analysis with current (Girardi et al. 2000; Yi et al. 2001) and custom-made stellar evolution models by the Yale and Padova groups will be presented in forthcoming papers, now in preparation.

\section{The cluster sample: Observations and data reduction}

Deep $V$ and $R$ images of SL 556, NGC 2155 and NGC 2173 were obtained at VLT-UT1 with FORS1 (Table 1), in service mode. These clusters were selected acording to a) their age (Sarajedini 1998; Corsi et al. 1994), b) their position in the galaxy, avoiding areas densely populated by LMC stars and c) their richness.

\begin{tabular}{lccr} 
Table 1. & \multicolumn{2}{l}{ Log of the observations } \\
\cline { 2 - 5 } Cluster & Seeing & \multicolumn{2}{c}{ Exposure Time (s) } \\
& & $V$ & $R$ \\
\cline { 2 - 5 } SL 556 & $0 .{ }^{\prime \prime}$ & 1864 & 1715 \\
NGC 2155 & $0.5-0.7$ & 1704 & 2235 \\
NGC 2173 & 0.5 & 928 & 855 \\
\hline
\end{tabular}

De-biasing and flatfielding were performed by means of the calibration frames (bias and sky flat fields) obtained during the same night, or one night before, of the target observations. The photometry was carried out with the DAOPHOTII/ALLFRAME package (Stetson 1994), following the steps described in Zoccali et al. (2001). The FORS1 $4 \times 4$ arcmin field of view allowed us to sample a region of the LMC field around each cluster. The photometry of this region was used to statistically decontaminate each cluster from field stars, taking into account the lower completeness of the more crowded regions, by means of artificial stars experiments.

\section{The Color-Magnitude diagrams}

Figure 1 shows the color-magnitude diagram (CMD) of the three clusters, decontaminated from field stars. The label in each panel indicate the annulus (in arcsecs) inside which we actually measured cluster stars: the innermost region 


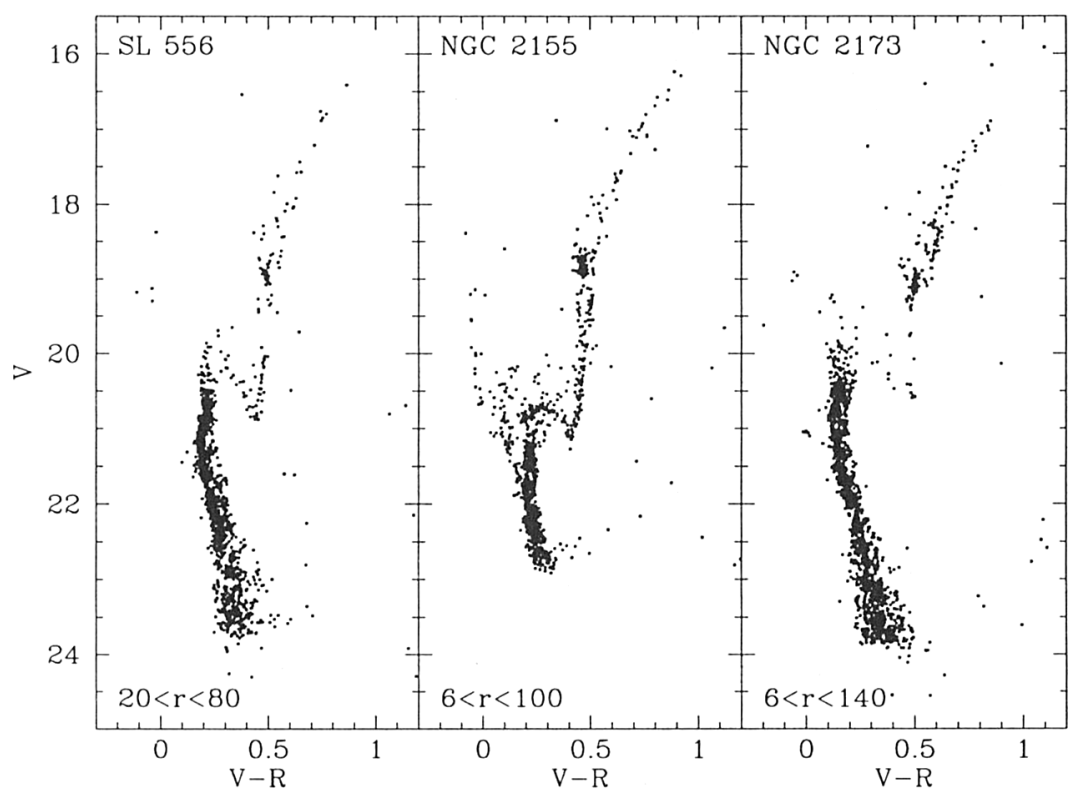

Figure 1. Decontaminated CMD of the target clusters.

was excluded because of its high crowding, while the outermost were taken as representative of the surrounding field population. Note that the different limiting magnitudes are mainly due to the presence of half moon in the night when NGC 2155 was observed, together with its worst seeing conditions.

\section{Future work}

The goal of our project is to produce a set of revised models for intermediate-age, low-metallicity stars, for both Yale and Padova libraries, through the comparison with these observed CMDs.

\section{References}

Girardi, L., Bressan, A., Bertelli, G., \& Chiosi, C. 2000, A\&AS, 141, 371

Corsi, C. E., Buonanno, R. Fusi Pecci, F., Ferraro, F. R., Testa, V., Greggio, L. 1994, MNRAS, 271, 385

Sarajedini, A. 1998, AJ, 116, 738

Yi, S., Demarque, P., Kim, Y-C., Lee, Y-W., Ree, C.H., Lejeune, T. \& Barnes S. 2001, ApJS, in press

Stetson, P.B. 1994, PASP, 106, 250

Zoccali, M., Renzini, A., Ortolani, S., Bica, E., Barbuy, B. 2001, AJ, 121, 2638 